\title{
miR-373-3p promotes lung adenocarcinoma cell proliferation via APP
}

\author{
XIAOXI FAN, SHUN XU and CHUNLU YANG \\ Department of Thoracic Surgery, First Affiliated Hospital of China Medical University, \\ Shenyang, Liaoning 110001, P.R. China
}

Received April 2, 2017; Accepted October 17, 2017

DOI: $10.3892 / \mathrm{ol} .2017 .7372$

\begin{abstract}
Previous studies have indicated that lung adenocarcinoma (LUAD) is one of the common human malignancies, and its incidence keeps rising. With the help of microarray technology, downregulation of miR-373-3p was observed in LUAD tissues compared with normal lung tissues. Notably, the present study demonstrated that the expression of amyloid precursor protein (APP) mRNA in LUAD tissues was overexpressed compared with adjacent tissues. Bioinformatic analysis demonstrated that miR-373-3p may interact with the $3^{\prime}$ untranslated region of APP mRNA, and then western blot and dual-luciferase reporter gene assays were employed to verify the interaction. Finally, CCK- 8 assays were used to measure the tumor-suppressing effect of miR-373-3p on A549 and it was demonstrated that overexpression of miR-373-3p may more effectively inhibit the proliferation of A549 compared with APP si-RNA. Overall, the findings suggest that miR-373-3p downregulation partly accounts for APP overexpression and leads to a promotion of cell growth in LUAD. miR-373-3p may therefore act as a valuable target in potential anticancer strategies to treat LUAD.
\end{abstract}

\section{Introduction}

Lung cancer is one of the most commonest malignant neoplasms among no matter men or women, and has the highest mortality (1). About 80 percent of all lung cancers are non-small cell lung cancer. Of those, $\sim 50 \%$ are lung adenocarcinoma (LUAD) (2). Hence, it is important to study the biological mechanism of LUAD, which are valuable to targeted therapy.

Correspondence to: Dr Chunlu Yang or Professor Shun Xu, Department of Thoracic Surgery, First Affiliated Hospital of China Medical University, 155 Nanjing North Street, Shenyang, Liaoning 110001, P.R. China

E-mail: clyang@cmu.edu.cn

E-mail: xushun610539@sina.com

Key words: miR-373-3p, APP, lung adenocarcinoma, proliferation
miRNAs play important roles in initiation and development of cancers, which suggest that they have potential to be targets for therapy in cancers (3). With the genetic sequencing technology developing, differentially expression miRNAs (DEMs) in LUAD tissues vs. noncancerous lung tissues have been detected (4). miR-373-3p is one of the members of miRNAs-371-372-373 family, which is transcribed from chromosome 19q13.42. Deregulation of miR-373-3p has been declared in many cancers, whether it acts as an oncogene or a tumor suppressor gene (5). Researches had proved that miR-373-3p has significant regulating function in breast cancer $(6,7)$, testicular germ cell tumors (8) and so on. Nevertheless, mechanism of miR-373-3p in LUAD has not been much clarified.

Amyloid precursor protein (APP) is a highly conserved type 1 transmembrane glycoprotein with a receptor-like structure and was considered to be closely related to the occurrence and progress of Alzheimer's disease (9). Meanwhile, APP has been shown up-regulated pathophysiologically in various kinds of cancers, including breast cancer (10), melanoma (11), and lung cancer (12). Bioinformatics analysis suggested that APP may be a target of miR-373-3p. In this study, the expression levels of APP and miR-373-3p in LUAD tissues vs. adjacent tissues were first time to be detected. Furthermore, using human LUAD cell line A549, the tumor-promoting effect of miR-373-3p in A549 was delineated then interaction between miRNA-373 and APP was identified to explore the in-depth mechanism.

\section{Materials and methods}

Bioinformatics analysis. The information of miRNA microarray was obtained from the NCBI GEO (Gene Expression Omnibus) under Platform GPL4717 and Series GSE18692. Detailed experimental process and design of the microarray was previously described by Puissegur et al (4). The differential expression of miRNAs (DEMs) was analyzed with the limma package. Under the condition of adjusted P-value $<0.01$ and $|\operatorname{logFC}|>2$, DEMs were considered to have significant differential expression between experiment groups and controls. The statistical tests were done by the R program version 3.2.2 (http://www.r-project. org/). To predict the target gene of miRNAs, 4 miRNA-target gene databases were searched including miRanda, RNA22Sites, TargetScan and picTarSites. 
Clinical specimen collection. 50 pairs of LUAD tissues and the adjacent nontumor lung tissues $(2 \mathrm{~cm}$ from the margin of the tumor), were collected from patients who operated at the First Affiliated Hospital of China Medical University (Shenyang, China) and we got approval from the Ethics Committee of the First Affiliated Hospital of China Medical University (IRB Approval 2012-40-2).

Cell culture and Cell transfection. Human LUAD cell lines A549, purchased from Shanghai Cell Bank of Chinese Academy of Sciences (Shanghai, China), were cultured in RPMI-1640 (Invitrogen, Carlsbad, CA, USA) medium supplemented with $10 \%$ fetal bovine serum (FBS; Invitrogen), $100 \mu \mathrm{g} / \mathrm{ml}$ streptomycin and $100 \mathrm{IU} / \mathrm{ml}$ penicillin. All the cells were maintained in a $37^{\circ} \mathrm{C}, 5 \% \mathrm{CO}_{2}$ incubator.

All the miRNA inhibitors, miRNA mimics, negative control (NC) of miRNA and siRNAs were chemically synthesized by Genepharma (Shanghai, China). All transfections in our study were transient and JetPRIME reagent (Polyplus-transfection) was added following the protocol. The cells were harvested for subsequent assays after RNA oligonucleotides successfully transfected for $48 \mathrm{~h}$.

$R N A$ isolation and quantitative reverse transcription-PCR ( $q R T-P C R)$. Total RNA was extracted from LUAD tissues, adjacent normal tissues and A549 by miRNeasy Mini kit (Qiagen, Hilden, Germany) according to the manufacturer's instructions. Complementary DNA (cDNA) of miR-373-3p was obtained with application of the QuantiMir RT kit (SBI). To verify the result of bioinformatics analysis, mRNA and miRNA of specimen were quantified by SYBR-Green qPCR Master Mix (Takara) through ABI 7500 Fast System thermocycler (Applied Biosystems Life Technologies, Foster City, CA, USA). Then, the qRT-PCR was applied to verify the transfection efficiency of oligonucleotides. The detection of miR-373-3p in detail was described by Zhang et al (13) and RUN6B (U6) was considered as an endogenous control. The primers of APP and $\beta$-actin are as followed: APP forward, GGAAGC GATGATAAGGTGGTAGAAGAACAA and reverse, CAT CACCATCATCATCGTCATCATCATCAG; $\beta$-actin forward, CCTTGCACATGCCGGAG and reverse, GCACAGAGCCTC GCCTT. All the experiments were performed in triplicate and data were calculated through $2^{-\Delta \Delta \mathrm{Ct}}$ method.

Protein extraction and western blotting. $48 \mathrm{~h}$ after transfection, proteins of the cell were extracted. Protein of the clinical sample and the cultured cell were separated by SDS-PAGE and transferred to nitrocellulose membranes, which were blocked with $5 \%$ fat-free milk for $1 \mathrm{~h}$. Then, the membranes incubated with anti-APP antibody (1:1,000; Abcam, Cambridge, UK) at $4^{\circ} \mathrm{C}$ overnight. Anti- $\beta$-actin antibody was served as the internal reference. The membranes were incubated with secondary antibodies for $30 \mathrm{~min}$ at room temperature after washing thoroughly. We detected the results by enhanced chemiluminescence technique (Amersham, Piscataway, NJ, USA) and quantified the level of expression of these proteins by application of Image $\mathbf{J}$ software.

Dual-luciferase assays. 3'UTR of the APP mRNA containing the potential target region for miR-373-3p were amplified by PCR. Overlap extension PCR was applied to amplify the mutant region of 3'UTR of the APP mRNA. Then, the region was cloned into the pmirGLO Dual-Luciferase miRNA Target Expression Vector (Promega Corp., Madison, WI, USA) and identified as dual-luciferase reporter vectors. The insertions were confirmed by Sangon Biotech (Shanghai, China) with commercial sequencing. The dual-luciferase reporter plasmids named pmirGLO-wt-APP and pmirGLO-mut-APP were co-transfected with miRNA mimics $(50 \mathrm{nM})$ or NC $(50 \mathrm{nM})$ using JetPRIME reagent (Polyplus-transfection). The cells were harvested after transfection for $48 \mathrm{~h}$. The luciferase activity was detected by the Dual-Luciferase ${ }^{\circledR}$ Reporter Assay System (Promega Corp.), according to the manufacturer's instructions.

Cell counting kit-8 (CCK-8) assays. After transfection $24 \mathrm{~h}$, A549 cells were seeded into 96 -well plates which density was $3-5 \times 10^{3}$ cells/well. At $0,24,48$, or $72 \mathrm{~h}, \mathrm{CCK}-8$ solution (Beyotime Biotech, Jiangsu, China) was added. The cells were cultured in a $37^{\circ} \mathrm{C}$ incubator for another $3 \mathrm{~h}$. To evaluate the number of viable cells, OD values at $450 \mathrm{~nm}$ wave-length (OD 450) were assessed daily.

Statistical analysis. All the statistical analyses except bioinformatics analysis were performed with SPSS 23.0 statistical software package (IBM SPSS, Armonk, NY, USA) and Graphpad prism 5 (GraphPad Software, Inc., La Jolla, CA, USA). Results were presented as mean $\pm \mathrm{SD}$ and $\mathrm{P}<0.05$ was considered to indicate a statistically significant difference.

\section{Results}

Identification of DEMs. A total of 6 DEMs were identified from GSE18692 datasets (GPL4717) on the basis of adj. $\mathrm{P}<0.01$ and $\mid \log \mathrm{FCl}>2$ (Fig. 1). Among them, 5 DEMs were significantly downregulation in LUAD, we selected miR-373-3p as our target.

miR-373-3p is downregulated and APP is overexpressed in LUAD tissues. In order to detect the expression levels of miR-373-3p and APP mRNA, qRT-PCR was used after the total RNA was extracted (Fig. 2). In comparison to the adjacent tissues, miR-373-3p expression was significantly downregulation in the LUAD tissue samples (Fig. 2A). In sharp contrast, the levels of APP mRNA were upregulation in the LUAD tissues compared with those in the adjacent normal tissues (Fig. 2B). Furthermore, we found that there is a negative relationship between miR-373-3p and APP mRNA $(\mathrm{r}=-0.4 ; \mathrm{P}<0.05)$ (Fig. 2C). Briefly, our results suggested that miR-373-3p may participate in the oncogenesis of LUAD partly through regulating APP, which has been reported as an oncogene in NSCLC.

miR-373-3p targets APP mRNA. A bioinformatic analysis using 4 miRNA-target gene databases showed that APP mRNA is a presumed target of miR-373-3p. The potential target region for miR-373-3p in the APP mRNA 3'UTR are showed in Fig. 3A. To verify that miR-373-3p can regulate APP mRNA, we investigated APP protein expression in A549 cells transfected with the miR-373-3p mimic. Successful transfection was confirmed by 


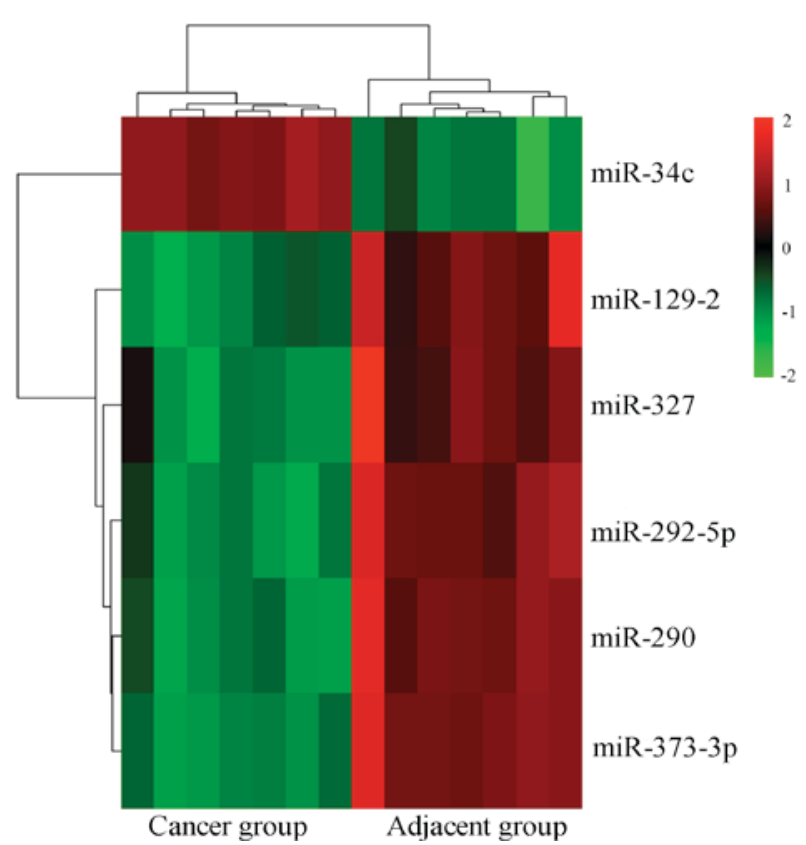

Figure 1. Cluster analysis for the differentially expressed miRNAs between lung adenocarcinoma tissues (cancer group) and adjacent tissues (normal group). 6 significant differential expressed miRNAs under the condition of adjusted $\mathrm{P}$-value $<0.01$ and $\log \mathrm{FCl}>2$ are showed in green (downregulation) and red (upregulation).
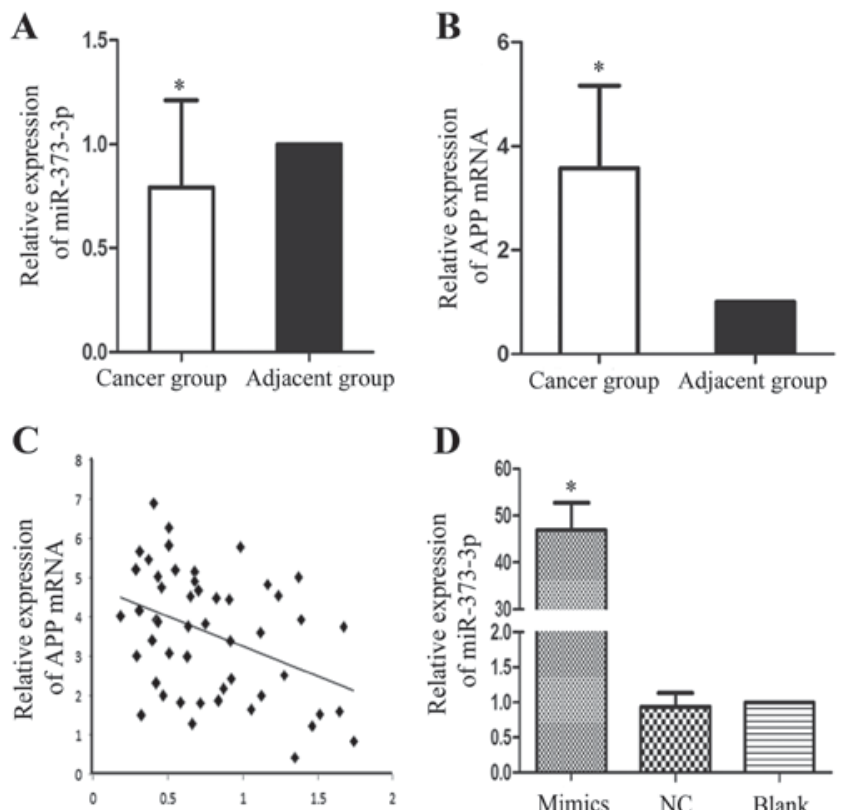

Relative expression of miR-373-3p

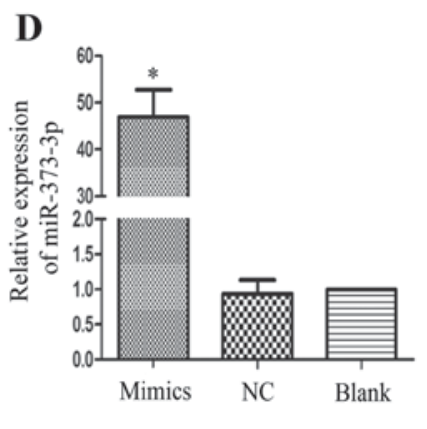

Figure 2. (A) The expression of miR-373-3p in cancer tissues was lower than that in normal tissues, ${ }^{*} \mathrm{P}<0.05$. (B) The expression of APP mRNA in in cancer tissues were significantly higher than that in normal tissues, ${ }^{*} \mathrm{P}<0.05$. (C) The relationship between the expression of miR-373-3p and APP mRNA. The expression of miR-373-3p was negatively associated with the expression of APP mRNA; $r=-0.4, P<0.05$. (D) the expression of miR-373-3p was evaluated by qRT-PCR in A549 cells $48 \mathrm{~h}$ after transfection to confirm transfection efficiency. The expression of miR-373-3p was markedly increase in mimics group compared with other groups. U6 was used as an internal control; ${ }^{*} \mathrm{P}<0.05$. APP, amyloid precursor protein; qRT-PCR, quantitative reverse transcription-PCR.

qRT-PCR (Fig. 2D). Western blotting results demonstrated that the expression of APP was markedly lower in the miR-373-3p group than in the blank or NC groups (Fig. 3B). Dual-luciferase reporter assays were implemented to validate if regulation exists. Dual-luciferase reporter vectors containing either the mutant or wild-type 3'UTR of APP mRNA was then constructed, and cotransfected into A549 cells together with the miR-373-3p mimic or NC. The results found that pmirGLO-wt-APP group was specifically responsive to miR-373-3p overexpression, but no significant difference was noticed between the relative luciferase activity of the miR-373-3p mimics group and that of cells cotransfected with NC in pmirGLO-mut-APP groups $(\mathrm{P}<0.05)$ (Fig. 3C). The results implied that miR-373-3p acts directly on the 3'-UTR of APP mRNA, and negatively regulates APP expression.

Forced expression of miR-373-3p inhibits the proliferation of LUAD cell line A549. To confirm the antitumor effect of miR-373-3p on LUAD, we synthesized a NC and a miR-373-3p mimic then introduced them respectively into the human LUAD cell line A549. The effects of miR-373-3p overexpression on LUAD cells were explored by CCK-8. In the CCK-8 assays, the miR-373-3p transfected cells showed slower proliferation trend than other groups (Fig. 4A). This indicates that the upregulation of miR-373-3p inhibits LUAD cell proliferation in vitro.

Overexpression of miR-373-3p more effectively inhibits the proliferation of A549 than APP si-RNA (si-APP). To compare the biological effect of miR-373-3p with that of si-APP on A549 behaviors, we used si-APP or miR-373-3p to silence APP expression, and the efficacy was verified with western blotting assays (Fig. 4B). The CCK-8 assays were performed to investigate the impact of miR-373-3p and si-APP in altering the growth trend of A549 cells. Both miR-373-3p and si-APP group showed proliferation-suppressing trend, but the proliferation trend was lower in miR-373-3p group (Fig. 4C). These data indicate that miR-373-3p had a stronger effect on A549 cells than si-APP, and miR-373-3p is a promising target for LUAD treatment since it affects more than one target mRNA and may involve in many regulatory network.

\section{Discussion}

Epidermal growth factor receptor (EGFR) inhibitors and ALK inhibitors have been applied in clinical practice for several years $(14,15)$, however, the patients with EGFR mutations or ALK gene translocations only account for a small part, which leads to the prognosis of LUAD patients has not been dramatically improved (16). Hence, it's necessary to develop more effective targeted therapies. With the help of genetic sequencing technology, we selected miR-373-3p, which is repressed in LUAD as our target. The expression of miR-373-3p is abnormal in various kinds of tumors, effecting process of proliferation, invasion and metastasis. miR-373-3p has been proved to be served as an oncogenic miRNA in testicular germ cell tumors by inhibiting LATS2, the tumor suppressor directly (8). A research suggested that expression of miR-373-3p are up-regulated in breast cancer, while invasion and metastasis of cancer cells could be promoted through down-regulation of CD44 expression (7). But the role of miR-373-3p remains controversial because later 
A

Position 709-715

of APP 3' UTR

5' - ...UCCCUGUUCAUUGUAAGCACUUU...-3'<smiles>C1=Cc2cc1c1cc2-1</smiles>

hsa-miR-373-3p 3'-...UGUGGGGUUUUAGCUUCGUGAAG...5',

B

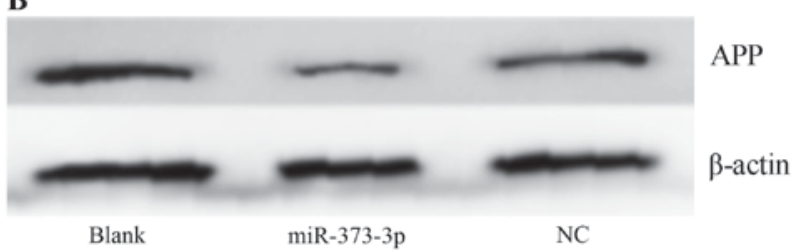

C

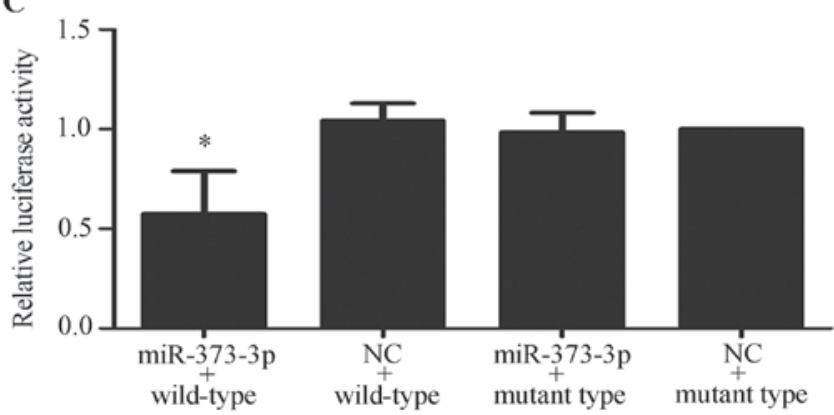

Figure 3. Interaction between miR-373-3p and APP mRNA. (A) Putative miR-373-3p binding sites in the 3'UTR of APP mRNA. (B) The effect of miRNA mimics on APP expression in A549 cells was confirmed by western blotting. The mimic group had elevated expression level of APP protein compared with other groups. $\beta$-actin was used as a loading control. (C) Dual luciferase reporter assays using vectors encoding putative miR-373-3p target sites in the 3'UTR of APP mRNA for either wild type or mutant type. Normalized data were calculated as Renilla/firefly luciferase activity. The relative luciferase activity was sharply lower in cells cotransfected with miR-373-3p mimic and pmirGLO-wt-APP, ${ }^{*} \mathrm{P}<0.05$ (The values for the pmirGLO-wt-APP + NC group compared with pRL-TK vector were set equal to 1). NC, negative control; APP, amyloid precursor protein.

then, the miR-520/373 family has been reported to serve as tumor suppressor in ER(-) breast cancer through linking the $\mathrm{NF}-\kappa \mathrm{B}$ pathways with TGF- $\beta$ pathways, and result in several consequences such as inflammation, tumor progression and dissemination (6). Recent reports demonstrated that miR-373-3p expression is silenced by histone modification in lung cancer cells, then promote proliferation, migration, and invasion of cancer cells via up-regulation of IRAK2 and LAMP1 target genes (17). Besides, there is very little study known about the relationship between miR-373-3p and LUAD.

A bioinformatic analysis suggested that APP mRNA may be a target of miR-373-3p, which aroused our interest to exploit the roles of these two molecules in LUAD. APP is a highly transmembrane glycoprotein and its expression is related with carcinogenesis of several tumors. For example, upexpression of APP is associated with greater metastatic tendencies as well as high motility and proliferation in breast cancer (10). A recent study conducted by Sobol et al (12) showed that decreased expression of APP leads to several consequences such as G0/G1 cell cycle arrest, decreased pRb phosphorylation, cell size abnormalities and necrosis in NSCLC. Furthermore, according to the analysis of 866 LUAD patients' overall survival rate by Kaplan-Meier plotter (http://www.kmplot. com/lung/), the elevated expression of APP may be relevant with poor prognosis (Fig. 5) (18).

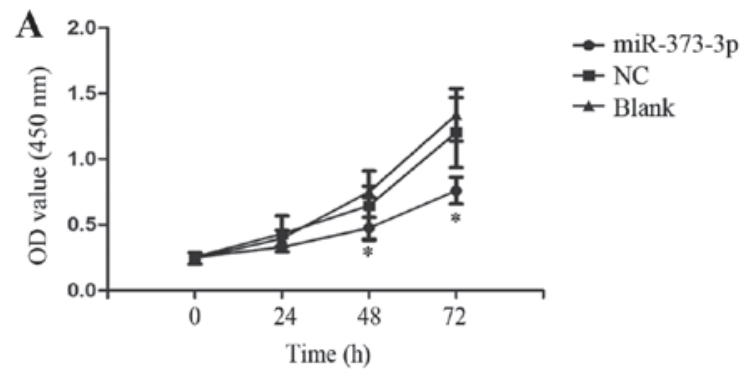

B
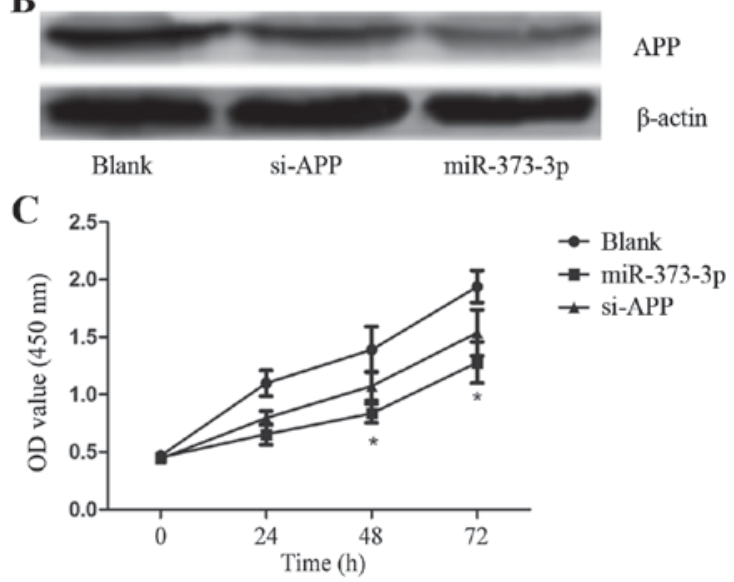

Figure 4. (A) Cell proliferation was detected by CCK-8 assays after transfection with miR-373-3p, which indicated miR-373-3p inhibit cell growth compared with the control groups, ${ }^{*} \mathrm{P}<0.05$. (B) miR-373-3p has a better silencing effect on APP than si-APP. (C) The proliferation rate of blank group was significantly higher than miR-373-3p and si-APP group. Both of them showed proliferation-suppressing trend, but the proliferation trend was lower in miR-373-3p group, ${ }^{*} \mathrm{P}<0.05$. si-APP, APP si-RNA; APP, amyloid precursor protein.

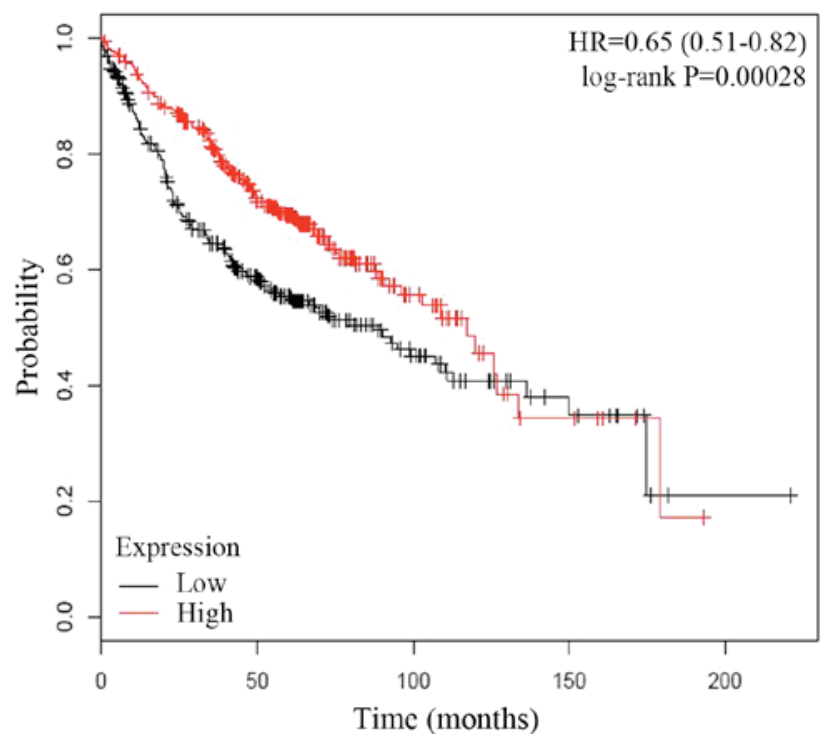

Figure 5. The Kaplan-Meier plotter downloaded from Kaplan-Meier plotter database (lung), $\mathrm{P}=0.00028<0.05$, which indicates elevated expression of APP may be related with poor prognosis. APP, amyloid precursor protein.

Taking these researches into consideration, we detected the miR-373-3p and APP mRNA expression in 50 paired tissues of LUAD patients. miR-373-3p was downregulation and APP mRNA was overexpression in their LUAD tissues compared with the adjacent normal tissues. Moreover, the 
expression of APP mRNA is negatively related with the expression of miR-373-3p. Through dual luciferase assays, the interaction between miR-373-3p and APP mRNA was verified. We then studied the effects of miR-373-3p on the cell growth of A549, and found that the upregulation of miR-373-3p inhibited cell proliferation by directly targeting APP mRNA. Furthermore, we observed that force expression of miR-373-3p had a stronger effect than APP siRNA on the behavior of LUAD cell line A549, which implies that miR-373-3p may target other oncogenes and is participate in more than one signaling pathway apart from this. Therefore, miR-373-3p could be a valuable target in LUAD treatment, and to achieve the goal, more LUAD patients and systematic follow-up should be included to make up for this study's deficiency. In addition, in vivo experiments and deeper exploration of the mechanisms of miR-373-3p/APP axis will also need to be exploited in the future.

In general, these data indicated miR-373-3p regulates the proliferation of LUAD partly via APP, and the expression of APP may be significantly related to the clinical outcomes of LUAD patients. These conclusions suggested that miR-373-3p may be a valuable target for potential anticancer strategy to treat LUAD.

\section{References}

1. Siegel RL, Miller KD and Jemal A: Cancer Statistics, 2017. CA Cancer J Clin 67: 7-30, 2017.

2. Diaz-Garcia CV, Agudo-López A, Pérez C, López-Martín JA, Rodríguez-Peralto JL, de Castro J, Cortijo A Martínez-Villanueva M, Iglesias L, García-Carbonero R, et al: DICER1, DROSHA and miRNAs in patients with non-small cell lung cancer: Implications for outcomes and histologic classification. Carcinogenesis 34: 1031-1038, 2013.

3. Kim VN and Nam JW: Genomics of microRNA. Trends in genetics 22: 165-173, 2006.

4. Puissegur MP, Mazure NM, Bertero T, Pradelli L, Grosso S, Robbe-Sermesant K, Maurin T, Lebrigand K, Cardinaud B, Hofman V, et al: miR-210 is overexpressed in late stages of lung cancer and mediates mitochondrial alterations associated with modulation of HIF-1 activity. Cell death and differentiation 18: 465-478, 2011

5. Wei F, Cao C, Xu X and Wang J: Diverse functions of miR-373 in cancer. J Transl Med 13: 162, 2015.

6. Keklikoglou I, Koerner C, Schmidt C, Zhang JD, Heckmann D, Shavinskaya A, Allgayer H, Gückel B, Fehm T, Schneeweiss A, et al: MicroRNA-520/373 family functions as a tumor suppressor in estrogen receptor negative breast cancer by targeting NF-kappaB and TGF-beta signaling pathways. Oncogene 31: 4150-4163, 2012.
7. Huang Q, Gumireddy K, Schrier M, le Sage C, Nagel R, Nair S, Egan DA, Li A, Huang G, Klein-Szanto AJ, et al: The microRNAs miR-373 and miR-520c promote tumour invasion and metastasis. Nat Cell Biol 10: 202-210, 2008.

8. Bing Z, Master SR, Tobias JW, Baldwin DA, Xu XW and Tomaszewski JE: MicroRNA expression profiles of seminoma from paraffin-embedded formalin-fixed tissue. Virchows Arch 461: 663-668, 2012.

9. Pandey P, Sliker B, Peters HL, Tuli A, Herskovitz J, Smits K, Purohit A, Singh RK, Dong J, Batra SK, et al: Amyloid precursor protein and amyloid precursor-like protein 2 in cancer. Oncotarget 7: 19430-19444, 2016.

10. Lim S, Yoo BK, Kim HS, Gilmore HL, Lee Y, Lee HP, Kim SJ, Letterio J and Lee HG: Amyloid-beta precursor protein promotes cell proliferation and motility of advanced breast cancer. BMC cancer 14: 928, 2014.

11. Botelho MG, Wang X, Arndt-Jovin DJ, Becker D and Jovin TM: Induction of terminal differentiation in melanoma cells on downregulation of beta-amyloid precursor protein. J Invest Dermatol 130: 1400-1410, 2010.

12. Sobol A, Galluzzo P, Weber MJ, Alani S and Bocchetta M: Depletion of amyloid precursor protein (APP) causes G0 arrest in non-small cell lung cancer (NSCLC) cells. J Cell Physiol 230: 1332-1341, 2015

13. Zhang Y, Zhao FJ, Chen LL, Wang LQ, Nephew KP, Wu YL and Zhang S: MiR-373 targeting of the Rab22a oncogene suppresses tumor invasion and metastasis in ovarian cancer. Oncotarget 5: 12291-12303, 2014

14. Xing RC, Zheng J, Zheng WH, Qin ZP, Liu W and Yao RC: Relevance of E-cadherin expression to EGFR-TKI molecular targeted therapy sensitivity/resistance and its clinical significance. Genet Mol Res 14: 5785-5792, 2015.

15. Steuer CE, Khuri FR and Ramalingam SS: The next generation of epidermal growth factor receptor tyrosine kinase inhibitors in the treatment of lung cancer. Cancer 121: E1-E6, 2015.

16. Ellis PM, Coakley N, Feld R, Kuruvilla S and Ung YC: Use of the epidermal growth factor receptor inhibitors gefitinib, erlotinib, afatinib, dacomitinib and icotinib in the treatment of non-small-cell lung cancer: A systematic review. Curr Oncol 22: e183-e215, 2015.

17. Seol HS, Akiyama Y, Shimada S, Lee HJ, Kim TI, Chun SM, Singh SR and Jang SJ: Epigenetic silencing of microRNA-373 to epithelial-mesenchymal transition in non-small cell lung cancer through IRAK2 and LAMP1 axes. Cancer Lett 353: 232-241, 2014.

18. Gyorffy B, Surowiak P, Budczies J and Lanczky A: Online survival analysis software to assess the prognostic value of biomarkers using transcriptomic data in non-small-cell lung cancer. PloS one 8: e82241, 2013. International (CC BY-NC-ND 4.0) License. 